\title{
Evaluation and audit in a paediatric disability service
}

\author{
Hilary D Cass, Barbara T Kugler
}

\begin{abstract}
Parental and professional responses to questionnaires evaluating a paediatric disability service are reported and the viability of auditing structural, process, and outcome aspects of clinical practice are discussed.

Expectations of waiting time to first appointment (met for only $52 \%$ of consumers) illustrate structural issues. Process issues are reflected in consumer reactions to outreach work (for example, $94 \%$ of parents and $84 \%$ of professionals found this supportive). Outcome measures such as consumer satisfaction with the service $(76 \%$ of consumers reported being 'very satisfied' and $20 \%$ 'fairly satisfied') suggest that service aims are being met.

Good concurrence of service aims with consumer needs is indicated by parental reasons for referral (for example, $\mathbf{7 5 \%}$ for diagnostic help, $73 \%$ for a better understanding of the disorder, $88 \%$ for practical help), referrers' reasons (for example, $55 \%$ for a second diagnostic opinion, $45 \%$ due to lack of local expertise), and reports from most other professionals involved with the case that a similar service was not provided locally. (Arch Dis Child 1993; 68: 379-383)
\end{abstract}

To survive in the NHS of the 1990 s, no service can depend solely on the commitment and calibre of its health care professionals; in a market driven system it is crucial that resources are managed efficiently to provide the optimum quality of care at costs that can be justified to purchasers.

Audit and quality assurance programmes provide invaluable contributory evidence to price and quality debates. These topics have now been widely reported ${ }^{1-4}$ but essentially focus on: (a) establishing the goals and objectives of the service concerned; (b) specifying standards of practice for the essential elements of service provision; and (c) organising a system whereby service performance can be monitored and compared with specified standards to direct necessary changes.

Harper House Children's Service, Horizon NHS Trust and Sub-
department of Paediatric Handicap, Department of Paediatrics, University College and Middlesex School of Medicine, School of London H D Cass B T Kugler

Correspondence to: Dr H D Cass, Harper House Children's Service, Harper Lane, Radlett, Herts WD7 7HU.

Accepted 25 August 1992 \section{patients' disabilities to allow them to enjoy the greatest possible independence within the con- straints of their impairment. For paediatric services an equally important goal is to support disability services easily measurable clinically. For example, the}

the disabled child's family in understanding and coming to terms with the child's difficulties. Unfortunately, long term benefits such as the degree of independence achieved in adulthood are not only difficult to measure but too remote to provide useful service feedback. Family well being, which reflects only indirectly on the index patient, is even more difficult to assess.

None the less, despite the fact that the impact of clinical intervention is qualitative rather than quantitative, it is still essential to set goals and standards that comprehensively reflect the specific concerns of purchasers, providers, and consumers and to find ways of assessing outcome.

One approach to the assessment of outcome is to develop techniques of assessment that demonstrate quality of care but do not demand clinically measurable variables. For example, patient satisfaction, in itself a non-clinical variable, can be used as a reflecton of outcome.

Alternatively, it is possible to audit aspects of clinical practice that strongly influence outcome and are more easily amenable to measurement. These can be divided into structural issues (that is, the availability and organisation of service resources) and process issues (that is, the way in which the patient is managed within the system from referral to discharge)..$^{46}$ For any service, structural data is usually readily available or easily collectable. Process issues, however, reflect the implementation of service goals and objectives and are closely associated with agreed guidelines for care ('best possible practice'). Hence carefully designed programmes of evaluation are needed.

Where indirect performance indicators are used to assess outcome, the audit process can become focused on specific performance targets. In maintaining quality it is equally important that the validity of the service goals and objectives should be subjected to review. The following essential facets of service quality, as described by Shaw, ${ }^{7}$ provide a useful framework for ensuring that the goals of the service remain pertinent and for assessing outcome: (a) appropriateness - the service or procedure is what the population or individual actually needs; (b) equity - a fair share for all the population; (c) accessibility - services are not compromised by undue limits of time or distance; (d) effectiveness - achieving the intended benefit for the individual and for the population); (e) acceptability - services are provided to satisfy the reasonable expectations of patients, providers, and community; and (f) efficiency - resources are not wasted on one service or patient to the detriment of another.

Which methods of evaluation are suitable for services in which there is difficulty in setting goals, specifying standards, and meaningfully assessing outcome? Faced with the need to 
obtain evidence on quality and effectiveness within the context of general purchaser-provider arrangements, our service (the Harper House Children's Service) set up a pilot evaluation study based on the use of consumer questionnaires. Examples from this study are used here to illustrate the issues discussed and to demonstrate that questionnaires can be used to evaluate crucial aspects of service delivery.

\section{Methods}

HARPER HOUSE CHILDRENS SERVICE

Harper House Children's Service is a tertiary specialist centre developed to facilitate care of disabled children in their local communities and receiving nationwide referrals. The service operates on an outreach basis focusing on children whose disorders have a low prevalence in the general population (for example, children with autism) but also receiving referrals of children with more commonly occurring disorders who are proving particularly difficult to manage locally.

The overall aim of the service is to assist in analysing a child's difficulties and skills, synthesising these findings, and formulating management plans. Within this framework, the service aims to see children as early as possible (to focus on primary disorders rather than secondary problems), to provide an appropriate multidisciplinary approach to children's difficulties, and to work in partnership with parents and local professionals.

Service provision is through initial appointments, multiprofessional assessment and investigation (clinic, home, and school based), direct therapeutic interventions (if these are feasible and appropriate), and follow up appointments. The service provided and the duration of involvement vary according to the specific needs of each child referred.

AIMS OF THE STUDY

These' were, firstly, to assess the validity of service objectives in terms of concurrence of service goals with consumer needs, and of whether alternative services could meet these goals more locally. Secondly, we aimed to assess consumer satisfaction with service standards reflecting structural issues (exemplified here by waiting times to first appointment); to assess consumer views on aspects of service delivery reflecting process issues (exemplified here by outreach work in the form of home and school visits); and to obtain outcome data on the service in terms of whether goals were achieved and to elicit other positive and negative outcomes for consumers (of use in directing service improvements).

Questionnaires were circulated to 50 randomly selected families and 50 randomly selected professionals ( 25 referrers and 25 professionals locally involved with the child but not responsible for the referral to Harper House) who had used the service in 1989.

They were posted in early November 1990 and collated in early December in preparation for a report to the regional health authority with a
January 1991 deadline. Neither parents nor professionals were contacted personally or 'prepared' in any way beforehand, and as all respondents were allowed to remain anonymous (to encourage unbiased responses), no 'follow up' procedures were instigated with respect to unreturned questionnaires.

Two main types of question design were used to elicit information on specific aspects of service delivery and general impressions of the service: (a) 'Forced choice' questions (usually also offering an option for free comment), for example, 'Would you encourage referrals to the Harper House Children's Service in future?' Answer: Yes/No/Unsure (professionals questionnaire); (b) 'Open ended' questions, for example, 'Is there anything that would make your role as referrer to Harper House easier or more satisfying?' (referrer's questionnaire).

An attempt was made not to tailor questions to bias answers: forced choice questions always included a negative pole, and, despite the ensuing difficulties for summarising data, most open ended questions were made deliberately non-specific.

\section{Results}

The overall response rate to the questionnaires at the time of analysis was a $79 \%$ return within one month (with comparable response rates for all three groups). By the end of February, the overall response rate had risen to $85 \%$. All the late returns reported themselves as satisfied with the overall service and gave responses in line with those analysed. Even if some of the remaining non-returns were attributable to dissatisfaction, the numbers would not be large enough to invalidate the results obtained.

Respondent anonymity did not appear to reduce returns and, interestingly, respondents who chose to identify themselves were as likely to do so when making negative comments as when making positive ones.

\section{ASSESSMENT OF THE VALIDITY OF SERVICE} OBJECTIVES

Concurrence of service goals with consumer needs

To assess the degree to which consumer requirements concurred with service aims, parents were asked to list the problems with which they most needed help at the time of referral. Multiple responses were obtained from nearly all families with $75 \%$ wanting diagnostic help, $73 \%$ specifically seeking a better understanding of their child's disorder (with regard to aetiology, course, and outcome with/without intervention), and $88 \%$ looking for practical help, advice, and support (from someone with experience of other children like their child). Thirty per cent of parents also reported being particularly concerned, at the time of referral, with seeking appropriate and comprehensive assessment of their child.

Asked why they had chosen to refer to Harper House, referrers also gave multiple responses. The two most common responses $(55 \%$ of referrers in each instance) were for a second opinion due to uncertainty over diagnosis, and previous 
experience of referring to Harper House. Lack of local expertise and acknowledgement of the skill and experience of specific members of the team were mentioned by 45 and $50 \%$ respectively. Forty five per cent made specific reference to school and home liaison work, $30 \%$ referred to access to a multidisciplinary team, and $30 \%$ to the desirability of multidisciplinary team assessment.

\section{Could goals be met by more local services?}

Given that referrers had initiated contact with the service and were therefore favourably biased respondents, other involved professionals were asked whether the service duplicated another locally based provision. Sixty eight per cent thought that Harper House had not duplicated a service available to them more locally; one professional felt there was 'some overlap with the School Psychological Service' on occasions and $26 \%$ felt unsure of the exact definition of 'duplication' as intended in this context, commenting, for example, that there may be 'some duplication in providing a second opinion, but this can be extremely reassuring for parents and professionals'.

These results suggest good concurrence of consumer requirements with service aims and provide evidence for appropriateness. A local child development team, skilled in the assessment of disabled children, adds to the understanding of the whole child but does not, in itself, provide or replace specialist, experience based knowledge of children with particular disorders. For children's needs to be met appropriately, both are necessary and the recognition of both by respondents to the questionnaires supports the validity of our service objectives. For disorders that occur only rarely in the general population, the specialist experience referred to cannot be obtained locally regardless of the excellence of any local multidisciplinary team. This must, by definition, rely on the centralisation of resources and on clinical experience of referrals drawn from a wide geographical area.

\section{ASSESSMENT OF CONSUMER SATISFACTION WITH OBTAINED STANDARDS OF WAITING TIMES TO FIRST APPOINTMENT}

Our specified standard for a desirable maximum waiting time to first appointment is four to six weeks. Unfortunately, the actual waiting time, both now and at the time of the study, is two to three months. As a result of this mismatch, we questioned parents, referrers, and other locally involved professionals on their expectations for waiting times. Figure 1 gives their responses.

An understanding of reasons for the delay was indicated by one local professional commenting, 'obviously I would prefer to have appointments sooner but I accept that this is not possible. If the Harper House Children's Service could be extended in order to cut down the waiting time I would be delighted'. In response to later questions on possible improvements to service provision, several parents, referrers, and nonreferring professionals highlighted shorter waiting times as a desirable improvement.

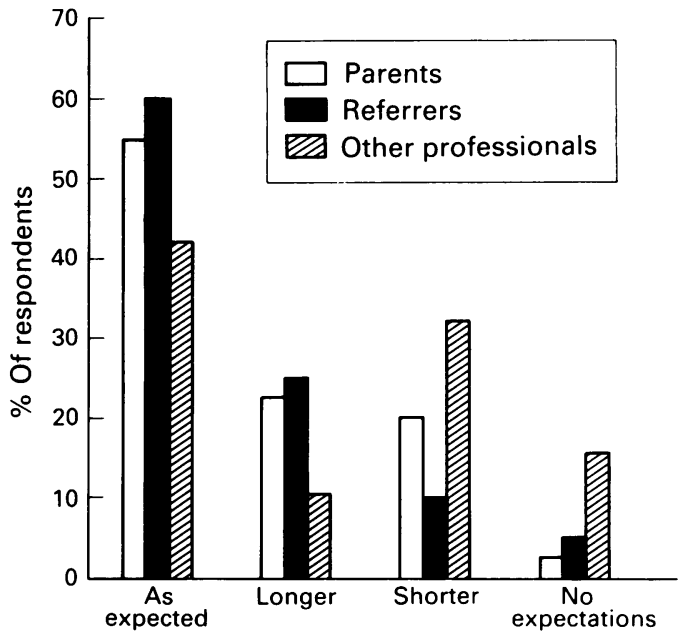

Figure 1 Expectations of waiting times of parents, referrers, and non-referring professionals.

Questions about waiting times provide data that allow evaluation of both accessibility (in terms of time) and acceptability (as shown by expectations). Although waiting times to first appointments are process issues related to the way referrals are managed by the service, evaluative data clearly cannot be interpreted without knowledge of structural issues. The duration of time that elapses between a referral being made and the referred child being seen is closely tied to staffing levels and the overall referral rates. Our own service, for example, saw a 53\% increase in referrals between 1985 and 1990, which was not mirrored by a corresponding increase in staffing levels.

For specialist services such as Harper House, consumer willingness to wait may well be related to the lack or shortage of alternative resources. As service providers, we feel that complacency at the lack of criticism in this situation is unwarranted.

\section{EVALUATION OF PROCESSES USED IN CLINICAL} ASSESSMENT

We see outreach work - meeting the child, parents, and professionals on their own ground as well as on our own clinic based ground - as an important means of achieving our aim of working in partnership with parents and local professionals. Parents' reactions to home visits and non-referring professionals' reactions to Harper House staff working on 'their patch' will therefore be used to illustrate the evaluation of one process used in clinical assessment. Responses from parents and non-referring professionals (who had experienced this form of service provision) were sought using forced choice questions that also offered an option for free comment. Figure 2 shows the forced choice responses (multiple).

The one parent who reported a home visit as 'anxiety provoking' explained 'I felt anxious after the visit because I was again forced into confronting a situation which one does try to suppress ... Things were said that I did not want to hear at that time'.

Three professionals reporting outside involve- 


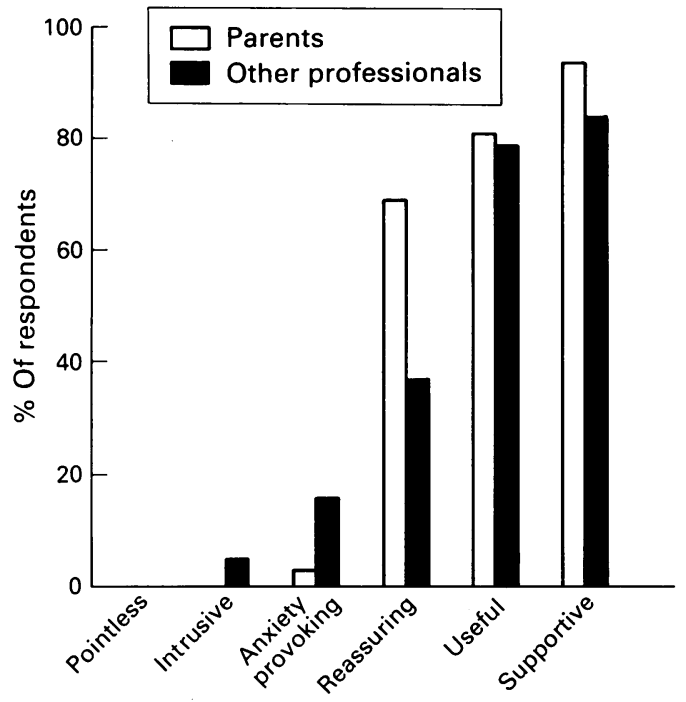

Figure 2 Parents' reactions to home visits and non-referring professionals' reactions to Harper House professionals working on 'their patch'.

ment as anxiety provoking gave as reasons, 'possible changes might result from visit', the family in question were 'playing professionals off against each other', and the involvement was 'unexpected'. The one professional reporting outside involvement as intrusive elaborated: 'intrusive initially, then supportive and useful ... Later I realised the intervention had helped to un-stick me at a point where I felt no change was possible'.

Further free comments were received from around two thirds of the parents and were relatively evenly split between (a) stressing this as a means of gaining more information and insight into the child and his problems, and (b) stressing the direct help received by child/ parents/whole family through this particular form of intervention.

Overall, these responses appear to indicate that inclusion of this outreach method of working in the process of clinical assessment and case management is perceived as effective by both parents and professionals.

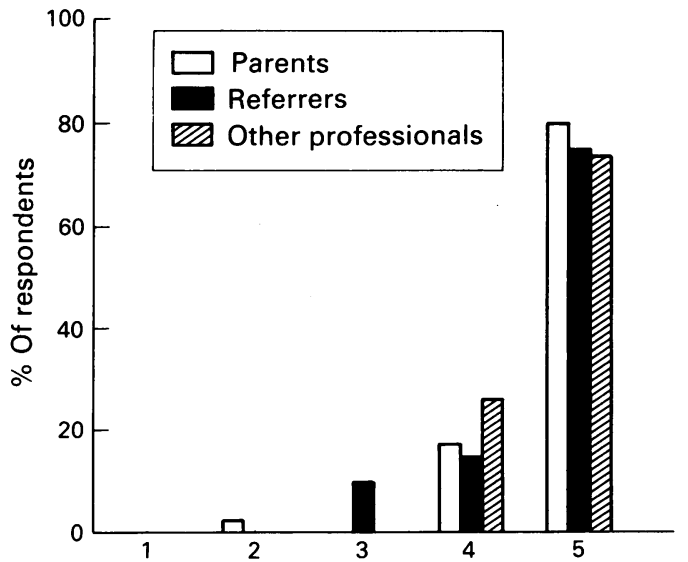

Figure 3 Satisfaction according to the following ratings: $1=$ very dissatisfied; 2 = fairly dissatisfied; $3=$ neither satisfied nor dissatisfied; $4=$ fairly satisfied; and $5=$ very satisfied.
EVALUATION OF OUTCOME

Given that consumer requirements are well in line with service goals, consumer satisfaction provides a useful indirect measure of whether goals are achieved. Estimations of overall satisfaction with the service were sought from all three consumer groups using a forced choice format with responses ranging from 'very dissatisfied' to 'very satisfied'. Figure 3 shows the responses of all three groups.

Other indicators of goal achievement includèd: whether consumers would use the service again for further problems or new referrals, or recommend it to others, or both; whether nonreferring local professionals would encourage potential future referrals from 'their patch' to Harper House; whether involvement with Harper House had positively affected the thinking and decision making of non-referring professionals; and whether referrers felt the service had been useful to themselves and the family they had referred. Positive responses to these questions were in excess of $85 \%$ and often reached $100 \%$.

The overall positive response rate to these outcome measures, in combination with a steadily rising referral rate, suggests that service goals are being met, and gives confidence that the service is not only appropriate, but also acceptable (expectations are satisfied) and effective (the intended benefit is achieved).

\section{Discussion}

The planning and provision of children's mental health services has recently received close scrutiny, with attempts made to specify the standards which patients and their families should expect, to turn overall policy objectives into service specifications, to pinpoint the essentials of service provision, and to encourage the monitoring of quality and outcomes. ${ }^{8}$ Maximum service effectiveness was seen as dependent on factors such as accurate assessment, diagnosis and investigation, and a multidisciplinary approach and sensitivity to the needs of families, but outcome measures were stated as imprecise. Hence clinical audit based on peer review, levels of patients' satisfaction, and the monitoring of data such as waiting list times or discharge letter delays was strongly recommended. Awareness of the relevance of these same issues to our own specialty prompted our use of consumer questionnaires in auditing the service.

The use of questionnaires and other patient satisfaction measures to assess quality of service delivery is well described. ${ }^{910}$ Criticisms levelled against the use of this technique raise concerns about respondent anonymity, the tailoring of question design to produce required answers, and the significance of non-returned questionnaires. It has been suggested that although consumer questionnaires have a part to play in quality assurance programmes, their contribution is relatively limited. ${ }^{2}$

Although this may be so for services where outcome is quantified by such objective measures as operative throughput, complications after an operation, or remission of symptoms, the situation is different in services where 
subjective improvements in quality of life are of crucial importance. In such instances even fairly unstructured interviewing has been reported as an effective means of evaluation."

In terms of the facets of service quality, information was elicited on four of Shaw's six points. ${ }^{7}$ Comparative data from other services and external review is necessary adequately to assess equity and efficiency. It is also clear that other techniques such as utilisation of an appropriate computer database to collect objective data (for example, the length of waiting times to initial appointment, or age on referral) are invaluable in helping to understand the viewpoints of purchasers, providers, and consumers.

Although we would not argue that questionnaires serve as an unsupported quality assurance programme, their value in combination with other techniques should not be underestimated. We believe that these examples show that they can be used to direct service goals and to aid in the process of setting realistic standards and assessing their achievement.
Our thanks to Gill Reding for her help in the preparation of the questionnaires and, along with other members of the Harper House Team, for help with their distribution and with data collation.

1 Clifford P, Leiper R, Lavender A, Pilling S. Assuring quality in mental health services - the Quartz system. London: RDP in association with Free Association Books, 1989.

2 Morgan J, Everett T. Introducing quality management in the NHS. International fournal of Health Care Quality Assurance NHS. Internation

3 Leahy A, Thambirajah M, Winkley L. Multidisciplinary audit in child and adolescent psychiatry. Psychol Bull 1992; 16: 214-5. 4 de Lacey G. What is audit? Why should we do doing it?
Hospital Update 1992: 458-66.

5 Donabedian A. Evaluating the quality of medical care. Millbank Memorial Fund $Q$ 1966; 44: 166-203.

6 Davison AJ. Action on audit. International fournal of Health Care Quality Assurance 1990; 3: 14-7.

7 Shaw C. Introducing quality assurance. London: King's Fund, 1986.

8 Kurtz $Z$, ed. With health in mind - mental health care for children and young people. London: Action for Sick Children in association with South West Thames Regional Health Authority, 1992.

9 Bishop F, Matthews F, Probert C, et al. Patients' views on how to run hospital outpatient clinics. $\mathcal{f} R$ Soc Med 1991; 84: to run

10 Damian $D$, Tattersall $M$. Letters to patients: improving communication in cancer care. Lancet 1991; 338: 923-5.

11 Brimblecombe F, Russell P. Honeylands - developing a service for families with handicapped children. London: National 